\title{
Characterization of dielectric charging in RF MEMS capacitive switches
}

\author{
R.W. Herfst ${ }^{1}$, H.G.A. Huizing ${ }^{1}$, P.G. Steeneken ${ }^{1}$, and J. Schmitz ${ }^{2}$ \\ ${ }^{1}$ Philips Research Laboratories Eindhoven \\ Prof. Holstlaan 4 (Postbox WAY41), 5656 AA Eindhoven, The Netherlands. \\ Phone: +31 (0)40 2745252 Fax: +31 (0)40 2744113. \\ E-mail: r.w.herfst@philips.com. \\ ${ }^{2}$ MESA+ Research Institute, Chair of Semiconductor Components, University of Twente. \\ P.O. Box 217, 7500 AE Enschede, The Netherlands.
}

\begin{abstract}
RF MEMS capacitive switches show great promise for use in wireless communication devices such as mobile phones, but the successful application of these switches is hindered by reliability concerns: charge injection in the dielectric layer ( $\mathrm{SiN})$ can cause irreversible stiction of the moving part of the switch.

We present a new way to characterize charge injection. By stressing the dielectric with electric fields on the order of 1 $M V / c m$, we inject charge in the dielectric, and use a new method to measure the effects it has on the $\mathrm{C}-\mathrm{V}$ curve. Instead of measuring the change in the pull-in voltage, this method measures the change in the voltage at which the capacitance is minimal. This way, no extra charge is injected during the measurement of the amount of injected charge, which reduces the effect it has on the tested switches, so that the effect of the intentionally induced stress voltage is not obscured by the measurement method.
\end{abstract}

\section{INTRODUCTION}

RF MEMS (Radio Frequency Micro-Electro-Mechanical Systems) capacitive switches show great potential for use in wireless communication devices such as mobile phones. This is due to the good RF characteristics and low power consumption of the switches [1]. Fig. 1 shows a schematic representation of an RF MEMS capacitive switch. The top electrode is suspended by tiny springs and can be pulled down by applying a voltage across the air gap between the two electrodes. Above a certain voltage, the balance between the attracting electrostatic force $F_{E}$ and restoring spring force $F_{\text {spring }}$ becomes unstable and the switch closes, which is marked by a sudden increase in the capacitance of the switch (Fig. 2). This voltage is called the pull-in voltage $V_{\mathrm{pi}}$. A dielectric layer prevents DC current flow. Once closed, the electric forces are much higher due to the shorter distance between the electrodes, and the switch will only open again if the voltage is lowered below the so-called pull-out voltage $\left(V_{\mathrm{po}}\right) . V_{\mathrm{pi}}$ and $V_{\text {po }}$ can be found by measuring the hysteresis present in the capacitance-voltage curve of the switch (Fig. 2). Since the electrostatic force is proportional to the voltage squared, pull-in and pull-out can happen for both positive and negative applied voltage, indicated in the figure with $V_{\mathrm{pi}}^{+}, V_{\mathrm{po}}^{+}$, $V_{\mathrm{pi}}^{-}$and $V_{\mathrm{po}}^{-}$.

In the closed state, the electric field in the dielectric layer is on the order of $1 \mathrm{MV} / \mathrm{cm}$. Because of this high field, charge

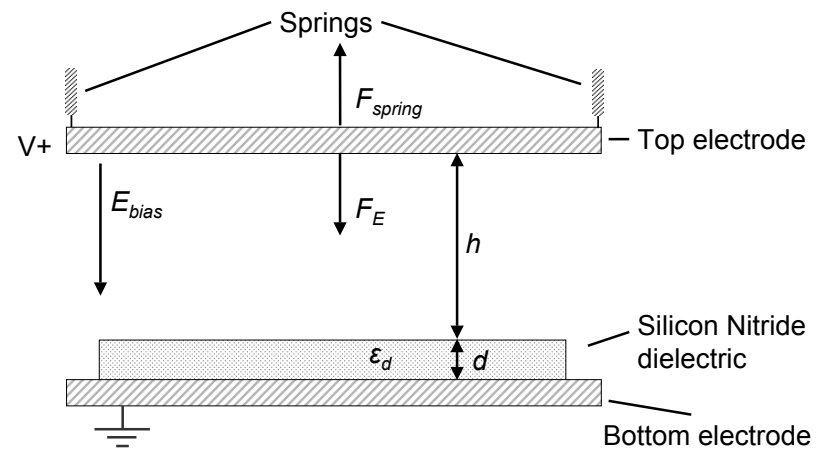

Fig. 1. Schematic representation of an RF MEMS. The top electrode of a parallel plate capacitor can be pulled down by applying a voltage greater than the pull-in voltage $\left(V>V_{\mathrm{pi}}\right)$, which is pulled up again by the springs if the voltage is lowered beneath the pull-out voltage $\left(V<V_{\text {po }}\right)$.

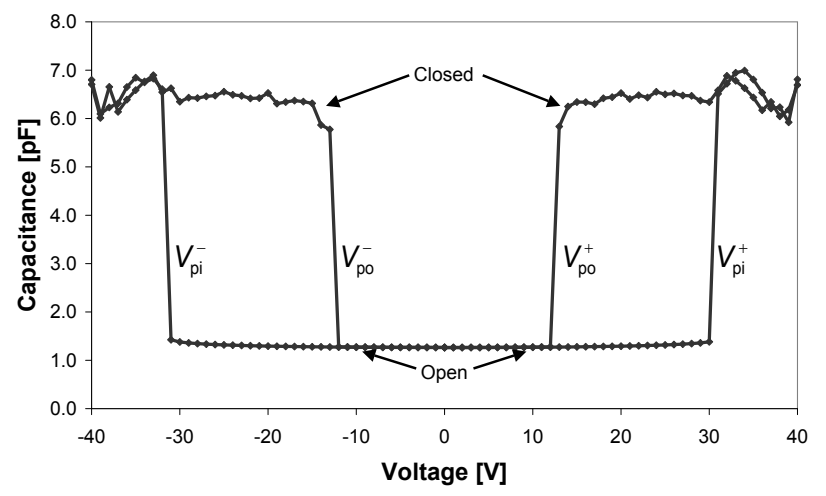

Fig. 2. Typical C-V curve of an RF MEMS capacitive switch. By ramping the voltage up, the top electrode is pulled down and the capacitance increases. Above $V=V_{\mathrm{pi}}$ the switch closes. When the voltage is ramped down again, the switch opens for $V<V_{\mathrm{po}}$.

is injected into the dielectric, which changes the electric field present in the gap between the two plates. This also changes the amount of charge in the bottom and top electrode, thereby influencing the electrostatic force (Fig. 3). The net effect of the superimposed E-field is a shift of the C-V curve [2], [3], which in turn affects the pull-in and pull-out voltages: $V_{\mathrm{pi}} \Rightarrow V_{\mathrm{pi}}+$ $V_{\text {shift }}$ and $V_{\text {po }} \Rightarrow V_{\text {po }}+V_{\text {shift }}$ (Fig. 4). $V_{\text {shift }}$ is proportional to the injected charge. A large amount of injected charge can 


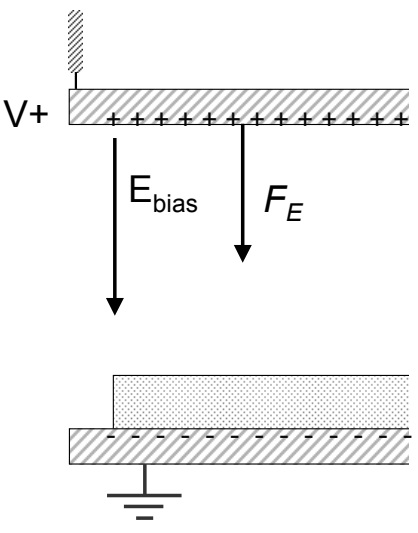

a)

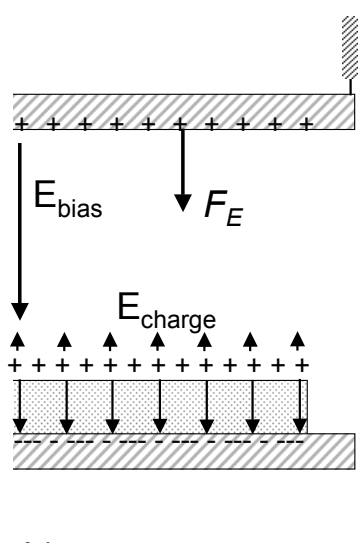

b)
Fig. 3. E-field and electrostatic force in a parallel plate capacitor. a) No fixed charges in the dielectric. b) Fixed surface charge in and on the dielectric.

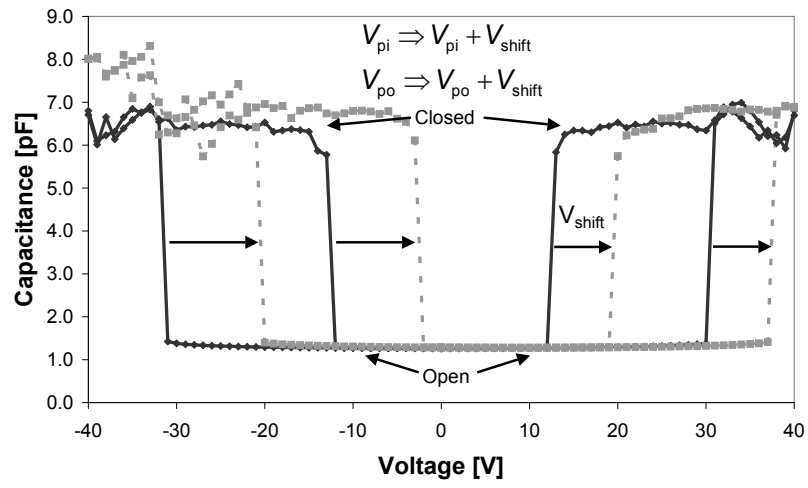

Fig. 4. C-V curve before (black) and after (grey) a switch has been stressed at 65 volt for 727 seconds.

even lead to failure of the switch due to stiction of the top electrode to the dielectric. This happens when $V_{\text {po }}^{-}$becomes positive or when $V_{\mathrm{po}}^{+}$becomes negative. In that case the switch can be in the closed branch of the hysteresis curve even at $0 \mathrm{~V}$. For instance, if $V_{\mathrm{po}}^{-}>0$, and the switch is closed by applying a voltage above $V_{\mathrm{pi}}^{+}$, it will not open again when the voltage is suddenly set to zero. Because of the inertia of the top electrode, the switch does not have enough time to open and skips the open branch of the hysteresis curve. Also, if $V_{\text {shift }}>0$, a higher actuation voltage is required to close the switch.

In this paper we consider three different methods for measuring the shift of the $\mathrm{C}-\mathrm{V}$ curve. Two are based on determining $V_{\mathrm{pi}}$ and one is based on determining the center shift of the C-V curve, which to our knowledge has not been proposed before. The influence that the measurements have on the switches is investigated. The new method, in contrast to other methods, does not degrade the switches and is subsequently used to characterize dielectric charging at several voltages.

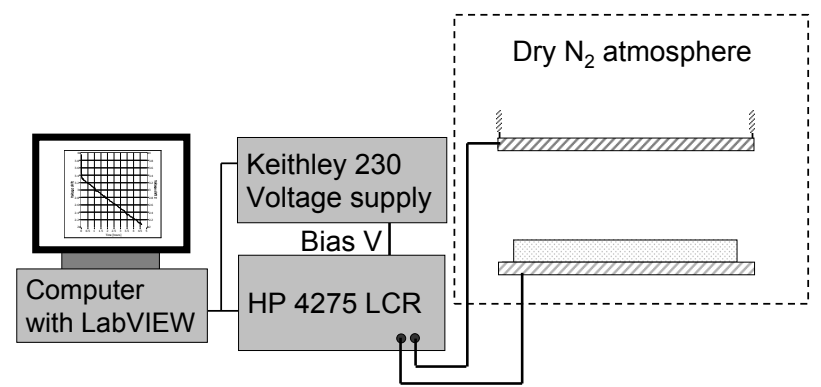

Fig. 5. Schematic view of the measurement setup. A LabVIEW computer program controls a HP4275 LCR meter and an Keithley 230 voltage Programmable Voltage Source. The LCR meter is connected to the RF MEMS capacitive switches, which are stressed and measured in a dry $\mathrm{N}_{2}$ atmosphere.

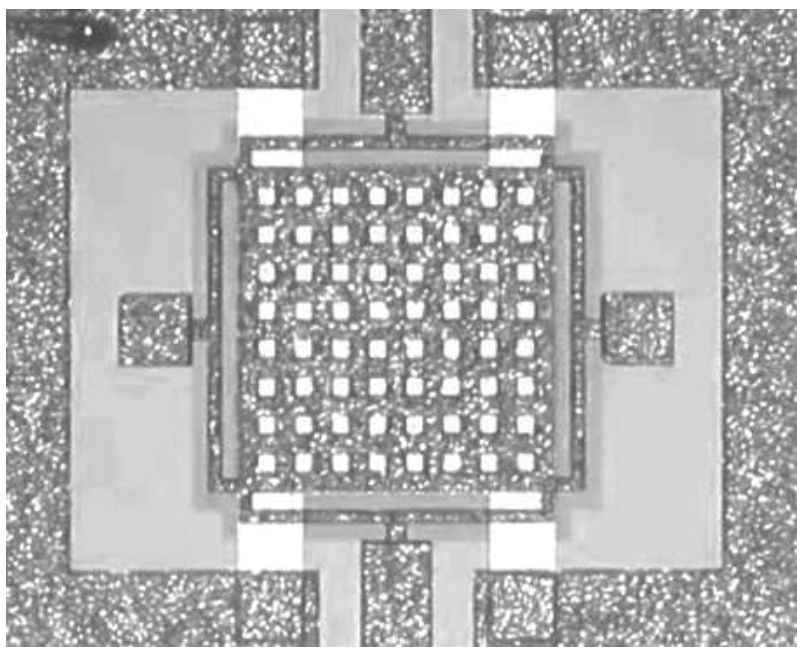

Fig. 6. Photograph of one of the switches used in the measurements. The top electrode of the device under study is $0.46 \times 0.46 \mathrm{~mm}^{2}$, the dielectric has a thickness of approximately $0.4 \mu \mathrm{m}$, and the air gap is approximately $5.2 \mu \mathrm{m}$.

\section{MEASURING DIELECTRIC CHARGES}

\section{A. Setup and measurement methods}

To study the charge injection, $V_{\text {shift }}$ is measured as a function of stress voltage and time. The setup with which these measurements are done is depicted schematically in Fig. 5. To avoid moisture from influencing the measurements, the switches are stressed and measured in a glovebox in a dry nitrogen environment at atmospheric pressure. A bias voltage is provided by a Keithley 230 Programmable Voltage Source to a HP4275 LCR meter which is then used to measure the capacitance as function of voltage. The layout of the switches which were used in the measurements is shown in Fig. 6.

A LabVIEW program was written to automate the measurements. It stresses the switch and periodically measures $V_{\text {shift }}$. After each period of stress, the shift of the C-V curve relative to the unstressed state must be measured. Several methods were considered:

1) Measure the $\mathrm{C}-\mathrm{V}$ curve with equidistant steps from below $-V_{\mathrm{pi}}$ to above $+V_{\mathrm{pi}}$ back to below $-V_{\mathrm{pi}}$ again. Compare to the original $\mathrm{C}-\mathrm{V}$ curve by determining the 
pull-in and pull-out voltages [4], [5]. $V_{\text {shift }}$ is determined by $V_{\text {shift }}=V_{\mathrm{pi}}-V_{\mathrm{pi}}^{t=0}$.

2) Similar to method 1, this method searches for the value of $V_{\mathrm{pi}}$ by an algorithm based on successive approximation. Note that after each guess for the pull-in voltage the switch must be allowed to open again. Again, $V_{\text {shift }}$ is determined by $V_{\text {shift }}=V_{\mathrm{pi}}-V_{\mathrm{pi}}^{t=0}$.

3) Only measure the shift of the center (opened switch) part of the $\mathrm{C}-\mathrm{V}$ curve, and estimate the voltage at which the capacitance has the lowest value. This is done by fitting a parabola $C(V)=a \cdot\left(V-V_{\text {shift }}\right)^{2}+C_{\text {open }}$ through the center of the $\mathrm{C}-\mathrm{V}$ curve (Fig. 7). Since the electrostatic force is proportional to $\left(V-V_{\text {shift }}\right)^{2}$ [6], $C(V)$ is symmetric around $V=V_{\text {shift }}$, therefore this fitted parabola accurately determines $V_{\text {shift }}$. To our knowledge, this is a new method, since another noncontact method [7] requires RF measurements.

Method 1 has the disadvantage that for each measurement of $V_{\text {shift }}$, the capacitance has to be measured at least $4 \times V_{\text {pi }} / V_{\text {step }}$ times, where $V_{\text {step }}$ is the resolution with which the $\mathrm{C}-\mathrm{V}$ curve is measured. Since the LCR meter takes roughly 1 second to accurately measure the capacitance, measuring $V_{\text {shift }}$ takes a significant amount of time so that during the measurement of $V_{\text {shift }}$ charge can leak away again. Also, voltages above $V_{\mathrm{pi}}$ have to be applied so that the switch 'sees' additional stress during the determination of $V_{\text {shift }}$. Method 2 is faster and also more accurate, but still has one of the problems of method 1: during the measurements voltages above $V_{\mathrm{pi}}$ have to be applied.

This leaves method 3, which is faster (in our case we measured the center part from the $\mathrm{C}-\mathrm{V}$ curve in 17 steps, significantly less than the 162 steps required to measure a complete $\mathrm{C}-\mathrm{V}$ curve from -40 to $+40 \mathrm{~V}$ with $1 \mathrm{~V}$ resolution, and also less than the 26 steps used for method 2) and has no risk of charging the dielectric further during the determination of $V_{\text {shift }}$. Also, since a large $V_{\text {shift }}$ may prevent the switch from opening at $0 \mathrm{~V}$, the measurement voltage is first set to the previous value of $V_{\text {shift }}$, rather than $0 \mathrm{~V}$. Due to this step, the switch will be forced into the open branch of the hysteresis curve and open. The new value of $V_{\text {shift }}$ is determined by measuring $C(V)$ around this previous measured value of $V_{\text {shift }}$. This way, $V_{\text {shift }}$ can even be measured when $V_{\text {po }}^{L}>0$ (or $V_{\mathrm{po}}^{R}<0$ ), thereby extending the range of voltage shifts that can be measured.

\section{B. Results: comparison of the measurement methods}

In this section we compare the influence of methods 1,2 and 3 on the measurement results. In Fig. 8 the effects and reproducibility of the determination of $V_{\text {shift }}$ with method 1, 2 and 3 are shown. No stress voltage was applied between consecutive measurements of $V_{\text {shift }}$. As can be seen, the measured values of $V_{\text {shift }}$ obtained by fitting a parabola (method 3 ) do only show a very slight upward trend (only $0.3 \mathrm{mV}$ per measurement), while the measured values of $V_{\text {shift }}$ obtained from the pull-in voltage (method 1 and 2) show a significant change when measured repeatedly. The noise on the $V_{\text {shift }}$ is

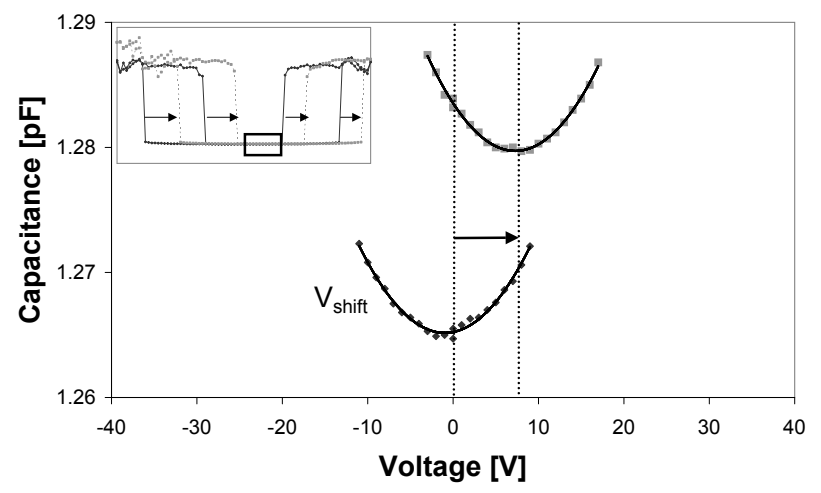

Fig. 7. Center of the C-V curve before (black) and after (grey) a switch has been stressed at 65 volt for 727 seconds. By fitting a parabola through the data, the center $V_{\text {shift }}$ can be accurately determined.

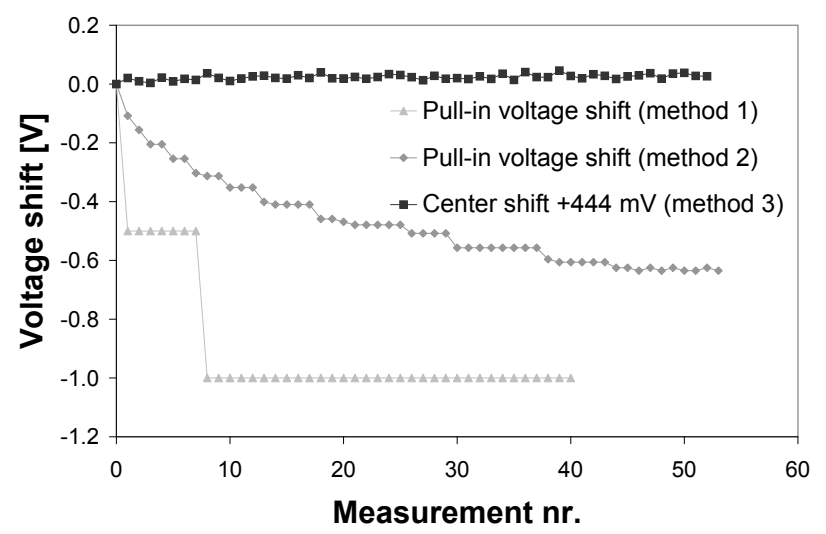

Fig. 8. Effect of measurements on the voltage shift determined by measuring the change in pull-in voltage and by determining the center of the $\mathrm{C}-\mathrm{V}$ curve when applying no stress voltage. All measurements are done on the same switch, first all center shifts, then the pull-in voltage shifts. Before the switch was stressed, an initial $V_{\text {shift }}$ of $-444 \mathrm{mV}$ measured. This was subtracted from the center shift results for better comparison with the two other methods.

also low with method 3 , the standard deviation with respect to the linear fit is $8.2 \mathrm{mV}$. The average drift of $V_{\text {shift }}$ obtained with method 2 is $-12 \mathrm{mV} /$ measurement, so that the drift is 40 times larger than the drift of the center shift method. Also the drift due to a single measurement with method 2 is larger than the standard deviation of $V_{\text {shift }}$ determined with method 3 . With method 1 this is even worse. This clearly shows the necessity to measure charging effects in the open state (method 3 ) to avoid that the measurement itself influences the result.

\section{Results: using method 3 to measure dielectric charging}

In Fig. 9 we have used method 3 to determine the shifts in the $\mathrm{C}-\mathrm{V}$ curve due to three different stress voltages. As one would expect, a higher stress voltage results in a faster and larger change of $V_{\text {shift }}$. According to Fig. 8, if these measurement had been done with method 2 instead of method 3 , a drift of about $-0.47 \mathrm{~V}$ would have been induced during the 20 measurements of $V_{\text {shift }}$ in 30 minutes. This would give a significant deviation: at $50 \mathrm{~V}$ the $\mathrm{C}-\mathrm{V}$ curve shifted between 1.0 and $1.8 \mathrm{~V}$ after 27 minutes. Even at $60 \mathrm{~V}$, the drift part would have been $7.5 \%$ of the measured value. With method 3 


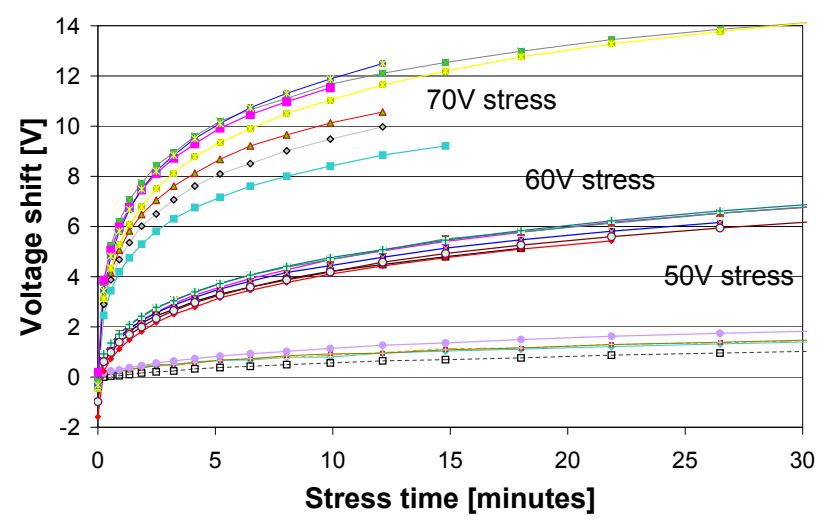

Fig. 9. Shift of $\mathrm{C}-\mathrm{V}$ curve as function of time at room temperature for 50 , 60 and $70 \mathrm{~V}$ stress. Spread in the measured results is higher at higher stress voltages.

a drift of only $6 \mathrm{mV}$ is expected. This indicates that the use of method 3 is much better suited to characterize charging, especially at lower stress voltages.

It is also clear from the measurements that at higher stress voltage the spread in the measured voltage shift curves becomes larger. It is speculated that this spread in the charging is due to variations in the thickness $d$ of the dielectric and surface roughness of the top electrode, which leads to a remaining air gap $h_{\text {closed }}$ between the top electrode and the dielectric in the closed state. The electric field is proportional to the applied voltage and inversely proportional to $\frac{d}{k}+h_{\text {closed, }}$, with $k$ being the relative dielectric constant. Variations in the nitride thickness and surface roughness lead to variations $\sigma_{E}$ in the electric field $E$ :

$$
\begin{aligned}
\sigma_{E} & =\left|\frac{\mathrm{d}}{\mathrm{d}\left(\frac{d}{k}+h_{\text {closed }}\right)} E\right| \sigma_{\frac{d}{k}+h_{\text {closed }}} \\
& =\left|\frac{V}{\left(\frac{d}{k}+h_{\text {closed }}\right)^{2}}\right| \sigma_{\frac{d}{k}+h_{\text {closed }}}
\end{aligned}
$$

The variations in $E$ are thus proportional to variations in $\frac{d}{k}+h_{\text {closed }}$ and to $E$ itself. Since the charging speed rapidly increases with $E$, the variations in $E$ translate to a larger spread in the charging curves at $70 \mathrm{~V}$.

\section{CONCLUSIONS}

The effects of charge injection in the dielectric layer of an RF MEMS capacitive switch was studied using a new measurement method which is both accurate and has less influence on the device under study than the commonly used procedure of measuring the change in the pull-in voltage. By measuring only the central part of the $\mathrm{C}-\mathrm{V}$ curve and fitting a parabola $C(V)=a \cdot\left(V-V_{\text {shift }}\right)^{2}+C_{\text {open }}$ to the data, $V_{\text {shift }}$ can be determined while the switch is in the open position, so that no additional charge is injected during the measurement, thereby separating the effect of intentionally induced stress and the stress effect that the measurement has on the device under test. This is especially important if dielectric charging at lower stress voltages is studied, due to the smaller and slower shifts at low stress voltages.

\section{ACKNOWLEDGMENT}

The authors wish to thank J.T.M. van Beek and M.J.E. Ulenaers for fabricating the RF MEMS capacitive switches.

\section{REFERENCES}

[1] Gabriel M. Rebeiz, chapter 1, page 1 RF MEMS - Theory, Design, and Technology, chapter 1, page 1, John Wiley \& Sons, Inc, 2003.

[2] E.K. Chan, K. Garikipati, and R.W. Dutton, Characterization of contact electromechanics through capacitance-voltage measurements and simulations, J. Microelectromechanical Systems, vol. 8, no. 2, 208-217 , June 1999

[3] W. Merlijn van Spengen, Robert Puers, Robert Mertens, and Ingrid De Wolf, A comprehensive model to predict the charging and reliability of capacitive RF MEMS switches, J. Micromech. Microeng. vol. 14, 514521, 2004.

[4] Xiaobin Yuan, James C.M. Hwang, David Forehand, and CHrles L. Goldsmith, Modeling and characterization of Dielectric-Charging Effects in RF MEMS Capacitive Switches, 2005 IEEE MTT-S International Microwave Symposium Digest.

[5] S. Mellé, D. De Conto, L. Mazenq, D. Dubuc, K. Grenier, L. Bary, O. Vendier, J.L. Muraro, J.L. Cazaux, and R. Plana, Modeling of the dielectric charging kinetic for capacitiv RF-MEMS, 2005 IEEE MTT-S International Microwave Symposium Digest.

[6] Xiaobin Yuan, Sergey Cherepko, James Hwang, Charles L. Goldsmith, Christopher Nordquist, and Christopher Dyck, Initial Observation and Analysis of Dielectric-Charging Effects on RF MEMS Capacitive Switches, 2004 IEEE MTT-S International Microwave Symposium Digest.

[7] J. Robert Reid, Richard T. Webster, and LaVern A. Starman, Noncontact Measurement of Charge Induced Voltage Shift in Capacitive MEMSwitches, IEEE Microwave and Wireless Components Letters, vol. 13, no. 9, september 2003. 